\title{
Parathyroid hormone-related protein induces cell survival in human renal cell carcinoma through the PI3K-Akt pathway: evidence for a critical role for integrin-linked kinase and nuclear factor kappa B
}

\author{
Abdelali Agouni ${ }^{1}$, Carole Sourbier ${ }^{1}$, Sabrina Danilin ${ }^{1}$, \\ Sylvie Rothhut ${ }^{1}$, Véronique Lindner ${ }^{1,2}$, Didier Jacqmin ${ }^{3}$, \\ Jean-Jacques Helwig ${ }^{1}$, Hervé Lang ${ }^{1,3}$ and Thierry \\ Massfelder ${ }^{1, *}$ \\ ${ }^{1}$ INSERM U727, Section of Renal Pharmacology and Physiopathology, \\ School of Medicine, University Louis Pasteur, Strasbourg 67085, France, \\ ${ }^{2}$ Department of Pathology and ${ }^{3}$ Department of Urology, Hôpitaux \\ Universitaires de Strasbourg, Strasbourg 67091, France \\ *To whom correspondence should be addressed. Tel: +33 3902434 56; \\ Fax: +33 3902434 59; \\ Email: thierry.massfelder@medecine.u-strasbg.fr
}

We have recently shown that parathyroid hormone-related protein (PTHrP), a cytokine-like polyprotein, is critical for human renal cell carcinoma ( $R C C)$ growth by inhibiting tumor cell apoptosis. Here, we have explored mechanisms by which PTHrP controls tumor cell survival. Using specific inhibitors of phosphoinositide 3-kinase (PI3K) and depletion of Akt kinase by RNA interference, we established that PTHrP is one of the main factor involved in the constitutive activation of this pathway in human RCC, independently of von Hippel-Lindau (VHL) tumor suppressor gene expression. Interestingly, PTHrP induced phosphorylation of Akt at $\mathrm{S} 473$ but had no influence on phosphorylation at T308. Through transfection with integrin-linked kinase (ILK) constructs and RNA interference, we provide evidence that ILK is involved in human RCC cell survival. PTHrP activates ILK which then acts as a phosphoinositide-dependent kinase (PDK)2 or a facilitator protein to phosphorylate Akt at S473. Among other kinases tested, only ILK was shown to exert this function in RCC. Using specific inhibitors, western blot and transcription assay, we identified nuclear factor kappa B (NF-кB) as the downstream Akt target regulated by PTHrP. Since $\mathrm{RCC}$ remains refractory to current therapies, our results establish that the $\mathrm{PI3K/ILK/Akt/NF- \kappa B}$ axis is a promising target for therapeutic intervention.

\section{Introduction}

Renal cell carcinoma (RCC), the major form of renal cancer, accounts for $3 \%$ of adult malignancy and is among the first 10 leading cause of cancer-related death worldwide (1-3). Its incidence is increasing steadily and worldwide each year; 200000 patients are diagnosed with this neoplasm, with an estimated 100000 deaths (3). The 5-year survival rate is $70 \%$ for localized RCC, but drops to $5 \%$ in the metastatic group. Metastatic RCC is resistant to radiotherapy and to systemic therapy including immunotherapy (1-3). The mechanisms of this resistance have not been elucidated. New therapeutic options for $\mathrm{RCC}$ are thus needed.

Biallelic inactivating mutations of the von Hippel-Lindau (VHL) tumor suppressor gene occur in patients with the VHL syndrome and in most patients with sporadic RCC (4-6). At the molecular level, the

\footnotetext{
Abbreviations: $\mathrm{Ab}$, antibody; $\mathrm{CRCC}$, conventional renal cell carcinoma; FACS, fluorescence-activated cell sorting; FKHR, forkhead transcription factor; GSK-3, glycogen synthase kinase-3; ILK, integrin-linked kinase; mTOR, mammalian target of rapamycine; NF- $\mathrm{BB}$, nuclear factor kappa B; PDK, phosphoinositide-dependent kinase; PKA, protein kinase A; PKC, protein kinase C; PI3K, phosphoinositide 3-kinase; PTHrP, parathyroid hormone-related protein; RCC, renal cell carcinoma; siRNA, small interfering RNA; TUNEL, Tdt-dependent dUTP-biotin nick end labeling; VHL, von Hippel-Lindau.
}

VHL gene products (pVHL) are involved in the degradation of hypoxiainduced transcription factors leading to the down-regulation of several hypoxia-induced transcription factor target genes including angiogenic and growth factors, such as vascular endothelial-derived growth factor and tumor growth factor- $\beta(7,8)$, that contribute to RCC tumorigenesis.

Parathyroid hormone-related protein (PTHrP), a polyprotein discovered in 1987, is the major causative agent in humoral hypercalcemia of malignancy associated to a broad range of tumors (3). However, the complex growth factor-like properties of PTHrP have shed new light onto potential roles of this peptide in the regulation of tumor growth and invasion (3).

Conventional renal cell carcinoma (CRCC) originates from the renal proximal tubular epithelium, a target tissue for PTHrP proliferation effects (9). We have recently shown that PTHrP, acting through its receptor PTH1R, is an essential growth factor for CRCC in vitro and in vivo and a new target for the VHL gene products $(10,11)$. However, the molecular mechanisms by which PTHrP exerts its effect on tumor cell survival were not investigated.

The phosphoinositide 3-kinase (PI3K)-Akt-signaling pathway is involved in many cellular processes including proliferation, death, migration and angiogenesis $(12,13)$. Once recruited at the plasma membrane through the activity of PI3K, the serine/threonine Akt kinase is activated by phosphorylation at two sites, T308 in the kinase domain and $\mathrm{S} 473$ in the regulatory tail, by phosphoinositidedependent kinase (PDK)-1 and PDK-2, respectively $(12,13)$. In contrast to PDK-1, the identity of PDK-2 is cell or tissue specific and its expression and activity are highly regulated (14). So far, almost 10 kinases have been suggested to function as a PDK-2 including integrin-linked kinase (ILK), protein kinase $\mathrm{C}$ (PKC), protein kinase A (PKA), double-stranded DNA-dependent protein kinase and rictormammalian target of rapamycine (mTOR) complex (14). Akt regulates the function of various proteins including the mTOR, the forkhead transcription factor (FKHR), glycogen synthase kinase-3 (GSK-3) and inhibitor of kappaB kinase $(12,13)$. The PI3K-Akt pathway is constitutively activated in various human cancers where it plays a critical role in tumor progression and in tumor resistance to therapies $(12,13)$. We recently showed that this pathway is constitutively activated in human CRCC independently of VHL expression and that it plays an essential role in CRCC progression through inhibition of tumor cell apoptosis (15).

Our objective was to identify the mechanisms by which PTHrP protects CRCC cells against apoptosis. Overall, our results uncover a new mechanism of regulation of cell apoptosis in human CRCC and suggest that the PI3K/ILK/Akt/nuclear factor kappa B (NF- $\kappa \mathrm{B})$ axis may be a promising target for therapy, regardless of VHL status.

\section{Materials and methods}

\section{Cell lines and cell culture}

Human CRCC cell lines Caki-1 (expresses VHL) and 786-0 (VHL deficient) (16) were obtained from American Type Culture Collection (Manassas, VA). Cells were maintained in Dulbecco's modified Eagle's medium (Invitrogen, Cergy-Pontoise, France) supplemented with $10 \%$ fetal bovine serum and used at $80 \%$ confluence, unless otherwise specified.

\section{Plasmids and stable transfection}

RCC cells (wild type) were grown until 50\% confluence and then stably transfected using Lipofectamine reagent (Invitrogen) according to the manufacturer's protocol with $10 \mu \mathrm{g}$ of pcDNA 3.1/His A, B and C vector alone or containing wild-type full-length ILK cDNA (ILK), kinase inactive ILK cDNA (in which S343 was replaced by A, iILK) or dominant-negative ILK cDNA (in which E359 was replaced by K, dnILK). All constructs were generously given by Prof. Shoukat Dedhar (Columbia Cancer Agency, Vancouver, British Columbia, Canada). Cells were grown in the presence of G418 $(500 \mu \mathrm{g} / \mathrm{ml})$. 
RNA interference and transient transfection

Small interfering RNA (siRNA) duplexes specific for human Akt1 (15) or human ILK were used to specifically knock down Akt and ILK, respectively. These siRNAs and control non-silencing siRNA were obtained from Ozyme (Cell Signaling local distributor, St Quentin Yvelines, France). Transient transfection of CRCC cells was performed according to the manufacturer's protocol. Briefly, cells were seeded in 12-well plates $(20000$ cells $/ \mathrm{ml})$, grown for $24 \mathrm{~h}$ (to reach $\sim 50 \%$ confluence) and then transiently transfected with $100 \mathrm{nM}$ Akt- or ILK-specific siRNA or control siRNA using the transfection reagent provided by the manufacturer, which also served as control without siRNA. Medium was replaced $24 \mathrm{~h}$ later by fresh medium and cells grown for an additional $24 \mathrm{~h}$ period ( $48 \mathrm{~h}$ post-transfection) prior to analysis.

\section{Immunoblotting and antibodies}

CRCC cells were treated as indicated in the Results, figures or figure legends Western blot analysis was performed as described previously (15). The following primary antibodies (Abs) (Ozyme) directed against proteins of human origin were used at the appropriate dilutions: polyclonal rabbit anti-Akt $\mathrm{Ab}$ at $1 / 250$ dilution; polyclonal rabbit anti-phospho-Akt (T308) Ab at 1/125 dilution; polyclonal rabbit anti-phospho-Akt (S473) at 1/250 dilution; monoclonal rabbit anti-GSK-3 $\beta \mathrm{Ab}$ at $1 / 250$ dilution and polyclonal rabbit anti-phosphoGSK-3 $\beta$ (S9) at $1 / 250$ dilution; polyclonal rabbit anti-cyclin D1 at $1 / 250$ dilution and polyclonal anti-phospho-cyclin D1 (T286) at 1/250 dilution; polyclonal rabbit anti-mTOR $\mathrm{Ab}$ at $1 / 250$ dilution and polyclonal rabbit antiphospho-mTOR (S2448) Ab at 1/250 dilution; polyclonal rabbit anti-FKHR $\mathrm{Ab}$ diluted at 1/250 and polyclonal rabbit anti-phospho-FKHR (S256) Ab diluted at $1 / 250$; polyclonal rabbit anti-ILK $\mathrm{Ab}$ at $1 / 250$ dilution and polyclonal rabbit anti-NF- $\kappa \mathrm{B}$ p65 $\mathrm{Ab}$ at $1 / 250$ dilution and polyclonal rabbit antiphospho-NF- $\kappa$ B p65 (S526) Ab at 1/250 dilution. For visualization of protein gel loading, a monoclonal mouse anti- $\beta$-actin Ab (Sigma-Aldrich, St Quentin Fallavier, France) was used at 1/5000 dilution. The appropriate horseradish peroxidase-conjugated secondary $\mathrm{Ab}$, i.e. donkey anti-rabbit Ab (Amersham, Courtaboeuf, France) at 1/1000 dilution or sheep anti-mouse Ab (Amersham) at $1 / 2000$ dilution was then used. Immunoreactivity was visualized with the ECL Western blotting detection kit (Amersham) and blots were exposed to Hyperfilm-ECL for up to $30 \mathrm{~min}$.

\section{Cell proliferation measurement}

CRCC cell proliferation was assessed by counting adherent cells, as described (15). CRCC cells were seeded in 24-well plates (20 000 cells $/ \mathrm{ml})$, grown for $48 \mathrm{~h}$ and then treated for $48 \mathrm{~h}$ with test substances at the concentrations indicated in the Results, figures or figure legends. Test substances included an affinity-purified polyclonal rabbit $\mathrm{Ab}$ directed against the $\mathrm{N}$-terminal region of PTHrP (PTHrP Ab, Bachem, Merseyside, UK), non-immune rabbit IgG (Sigma-Aldrich), LY294002 (Sigma-Aldrich), wortmannin (VWR International, Strasbourg, France), BAY 11-7085 (VWR International), calphostin C (Sigma-Aldrich), U73122 (VWR International), phorbol 12-myristate 13acetate (Sigma-Aldrich), inactive $4 \alpha$-phorbol 12-myristate 13-acetate (SigmaAldrich), 2' ,5' -dideoxyadenosine (Sigma-Aldrich), H89 (VWR International) and double-stranded DNA-dependent protein kinase inhibitor (SigmaAldrich).

\section{Tdt-dependent dUTP-biotin nick end labeling staining}

CRCC cells were seeded in four-well Tissue-Tek chamber slides (Deutscher, Brumath, France) at 20000 cells per well, grown for $48 \mathrm{~h}$ and then treated for $48 \mathrm{~h}$ with PTHrP Ab $(5 \mu \mathrm{g} / \mathrm{ml})$ or PTHrP diluent (water, control). The concentration of the PTHrP Ab was chosen from our previous studies $(10,11)$ to give a submaximal $50 \%$ decrease in cell viability. Cell death was measured using the cell death detection kit based on the Tdt-dependent dUTP-biotin nick end labeling (TUNEL) method, according to the manufacturer's protocol (Roche Diagnostics, Meylan, France) and as described previously (15). Total and stained cells were counted in 10 fields $\left(0.25 \mathrm{~mm}^{2}\right.$ each), and cell death was expressed as a percentage of stained cells to total cells.

Fluorescence-activated cell sorting analysis

CRCC cell apoptosis was assessed by fluorescence-activated cell sorter analysis. CRCC cells were seeded in $25 \mathrm{~cm}^{2}$ plates $(20000$ cells $/ \mathrm{ml})$, grown for $48 \mathrm{~h}$ and then treated for $24 \mathrm{~h}$ with PTHrP Ab $(5 \mu \mathrm{g} / \mathrm{ml})$ or PTHrP diluent (water, control) prior to fluorescence-activated cell sorting (FACS) analysis. In an other set of experiment, cells transiently transfected with siRNA or control siRNA were subjected to FACS analysis $96 \mathrm{~h}$ after transfection. Briefly, floating and adherent cells were harvested by trypsinization and were re-suspended in $100 \mu \mathrm{l}$ of incubation buffer $\left(140 \mathrm{mM} \mathrm{NaCl}, 5 \mathrm{mM} \mathrm{CaCl}_{2}\right.$ and $10 \mathrm{mM} \mathrm{N}-2$ hydroxyethylpiperazine- $N^{\prime}$-2-ethanesulfonic acid) containing Annexin V-FITC and propidium iodide $(1 \mu \mathrm{g} / \mathrm{ml})$. The mixture was then incubated in dark chamber at $4^{\circ} \mathrm{C}$ for $10 \mathrm{~min}$. After centrifugation, cells were fixed in dark chamber in $200 \mu \mathrm{l}$ of formol $1 \%$ at $4^{\circ} \mathrm{C}$ for $10 \mathrm{~min}$. Cells were then centrifuged, re-suspended in $200 \mu \mathrm{l}$ of incubation buffer and subjected to fluorescenceactivated cell sorter Scan (Becton Dickinson, Franklin Lakes, NJ) analysis using the corresponding software. Cell apoptosis was then expressed as apoptotic cells in percent of total cells. The use of Annexin $\mathrm{V}$ and propidium iodide together allows to assess both apoptosis and necrosis at the same time.

\section{$N F-\kappa B$ transcriptional activity assay}

$\mathrm{NF}-\kappa \mathrm{B}$ transcriptional activity was assayed using a non-radioactive NF- $\kappa \mathrm{B}$ p50/p65 transcription factor assay (Chemicon, Hampshire, UK) that combines electrophoretic mobility shift assay with the enzyme-linked immunosorbent assay to measure transcription activity in nuclear extracts. Preparation of samples and assay procedures were performed according to the manufacturer's protocol. Briefly, CRCC cells were seeded in $25 \mathrm{~cm}^{2}$ plates $(20000$ cells/ $\mathrm{ml})$, grown for $48 \mathrm{~h}$ and then treated for 2 or $5 \mathrm{~h}$ with PTHrP Ab $(5 \mu \mathrm{g} / \mathrm{ml})$ or PTHrP diluent (water, control). Nuclear extracts were prepared using the nuclear extraction kit (Chemicon) exactly following the kit's protocol. Nuclear protein concentrations were determined according to the method of Lowry et al. (17) and nuclear extracts were frozen at $-80^{\circ} \mathrm{C}$ until transcription assay. Nuclear extracts were then mixed with a biotinylated oligonucleotide containing the consensus DNA-binding sequence of NF- $\kappa \mathrm{B}$. The bound NF- $\kappa \mathrm{B}$ transcription factor subunits were then revealed with specific rabbit anti-NF- $\kappa \mathrm{B}$ p50 and anti-NF- $\kappa$ B p65 Abs. A positive cell extract, a non-specific doublestranded oligonucleotide and a specific competitor double-stranded oligonucleotide are used as controls. Specific binding is detected colorimetrically at $450 \mathrm{nM}$ (reference wavelength $650 \mathrm{~nm}$ ) using the $\mu$ Quant multiplate reader (Fisher Bioblock Scientific, Illkirch, France).

\section{Statistical analysis}

All values are expressed as mean \pm SEM. Values were compared using multifactorial analysis of variance followed by the Student-Newman-Keul's test for multiple comparisons. $P<0.05$ was considered significant.

\section{Results}

PTHrP induces cell survival through inhibition of tumor cell apoptosis

Both cell lines used here, i.e. 786-0 and Caki- 1 cells, produced PTHrP at maximally effective concentrations $(10,11)$. Thus, all the study was performed using a polyclonal anti-PTHrP Ab directed against the N-terminal part of the PTHrP molecule that we used previously $(10,11)$. Exposure of cells to PTHrP Ab for $48 \mathrm{~h}$ decreased cell density by $40-50 \%$ in both cell lines (Figure 1A), as expected from our previous studies $(10,11)$. Non-immune $\operatorname{IgG}$ was not included in these experiments since no effect was observed on human CRCC cell growth in our same previous studies. The effect was obtained through induction of cell apoptosis in both cell lines as demonstrated by TUNEL staining (Figure 1B) and FACS analysis (Figure 1C). No evidence of cell necrosis was noticed in response to PTHrP Ab (data not shown). Since the decrease in cell density was due to cell apoptosis, the following experiments dealing with cell growth were analyzed by measuring cell density.

\section{PTHrP induces cell survival through stimulation of the PI3K-Akt} pathway

We recently showed that the PI3K-Akt-signaling pathway is constitutively activated in human CRCC and that it plays a critical role in the growth of these tumors $(3,15)$. We thus investigated whether PTHrP activates this pathway in human CRCC. Phosphorylation of Akt at both T308 and S473 was substantially decreased by exposing cells for $2 \mathrm{~h}$ to LY294002 and wortmannin, two PI3K inhibitors $(18,19)$ (Figure 2A). As expected from our previous study $(3,15)$, treatment of 786-0 cells with either LY294002 or wortmannin for $48 \mathrm{~h}$ decreased cell density up to $80 \%$ in a concentration-dependent manner (Figure 2B). Similar effects were observed in Caki-1 cells (data not shown). In 786-0 cells, both PI3K inhibitors inhibited cell death induced by PTHrP Ab by up to $90 \%$ in a concentrationdependent manner, suggesting that endogenous PTHrP acts mainly through the PI3K-Akt-signaling pathway (Figure $2 \mathrm{C}$ ). PTHrP Ab remaining effect represents the difference of the apoptotic effect of the PTHrP Ab alone with its effect in the presence of the inhibitor at 

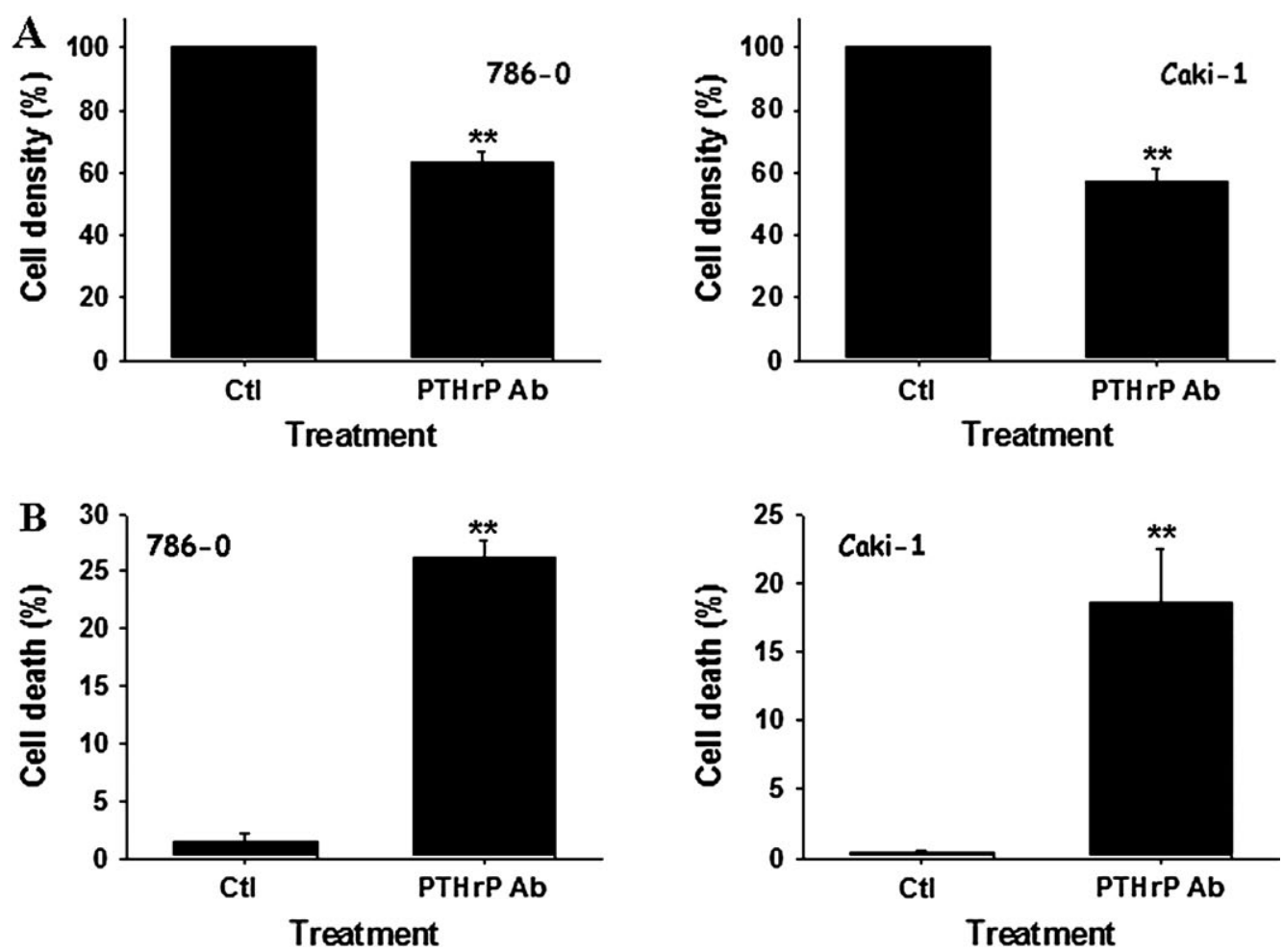

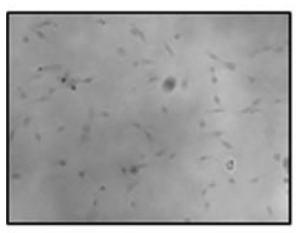

Ctl

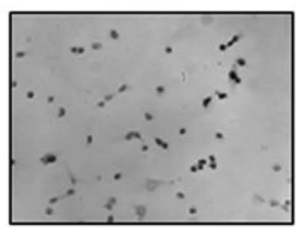

PTHrP Ab

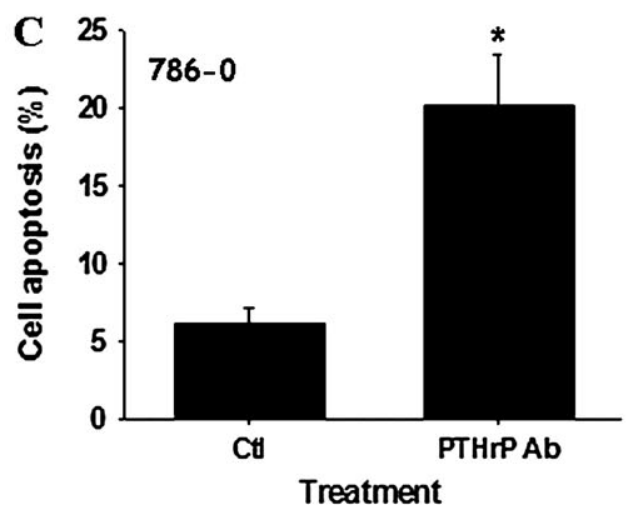

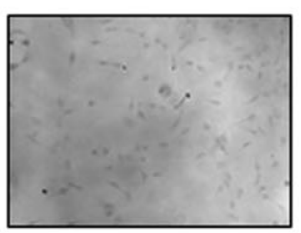

Ctl

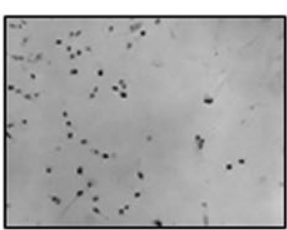

PTHrP Ab

Fig. 1. PTHrP induces cell apoptosis in human CRCC cell lines. (A) Effects of PTHrP blockade with PTHrP Ab (5 $\mu \mathrm{g} / \mathrm{ml})$ on cell density in $786-0$ cells (left panel) and Caki-1 cells (right panel). Results are shown as mean \pm SEM., $n=4$ (786-0 cells) and $n=13$ (Caki-1 cells); ${ }^{* *} P<0.01$ from control (Ctl). (B) Effects of PTHrP blockade with PTHrP Ab $(5 \mu \mathrm{g} / \mathrm{ml})$ on cell death as measured by TUNEL staining in 786-0 cells (left panel) and Caki-1 cells (right panel). Results are shown as mean \pm SEM, $n=4$ (786-0 cells) and $n=4$ (Caki-1 cells); ${ }^{* *} P<0.01$ from control. Photographs show TUNEL staining of 786-0 cells (two left photographs) or Caki-1 cells (two right photographs) treated in control or with PTHrP Ab (right) (magnification $\times 200$ ). Photographs shown are representative fields of at least three independent experiments. (C) Effects of PTHrP blockade with PTHrP Ab (5 $\mu \mathrm{g} / \mathrm{ml})$ on cell apoptosis as assayed by FACS in $786-0$ cells (left panel) and Caki-1 cells (right panel). Results are shown as mean \pm SEM, $n=7-12$ (786-0 cells) and $n=3$ (Caki-1 cells); ${ }^{*} P<0.05$ from control.

various concentrations. The remaining effect is then expressed in percentage calculated from the effect of the PTHrP Ab alone. In following results, where PTHrP Ab remaining effects are depicted, similar calculations were performed. Precisions are given in the Figure legends. Again, same effects were observed in Caki-1 cells (data not shown). In the presence of Akt-specific siRNA, Akt expression in $786-0$ cells was decreased by $\sim 50 \%$ as assessed $24 \mathrm{~h}$ after transfection
(Figure 2D, blot). After $96 \mathrm{~h}$ post-transfection, cell density was decreased by $70 \%$ (Figure 2D, left panel). The effect of PTHrP Ab, added $48 \mathrm{~h}$ after transfection for an additional $48 \mathrm{~h}$ period, was decreased by $90 \%$ in the presence of Akt-specific siRNA (Figure 2D, right panel) suggesting that PTHrP exerts its survival effect mainly through the Akt-signaling pathway. Similar results were obtained in Caki-1 cells (data not shown). 
A

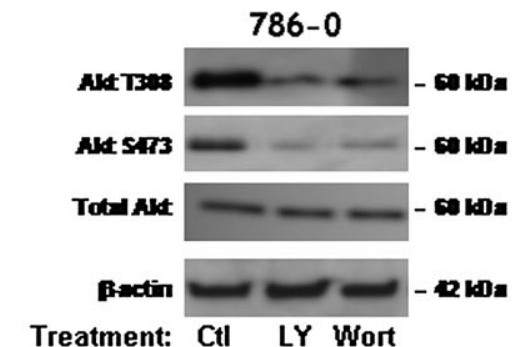

B

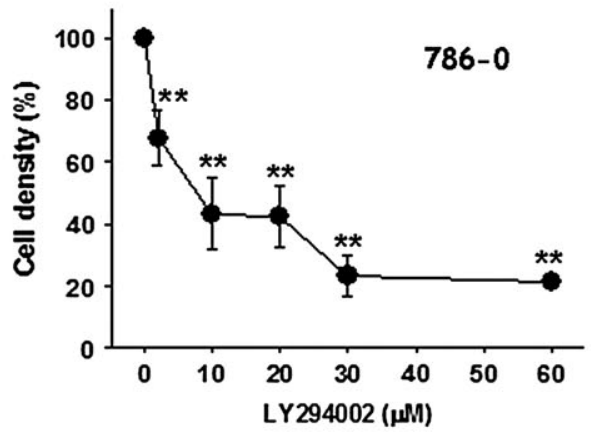

C

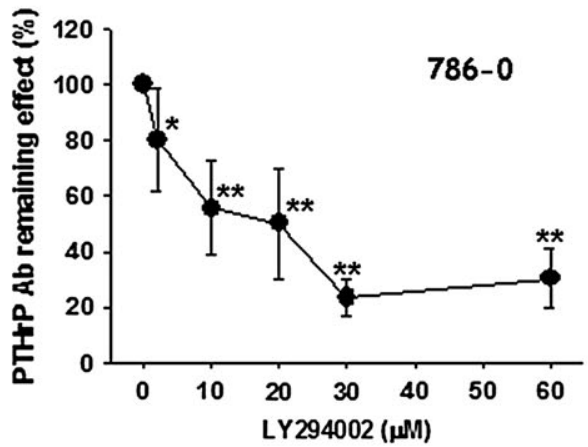

Caki-1
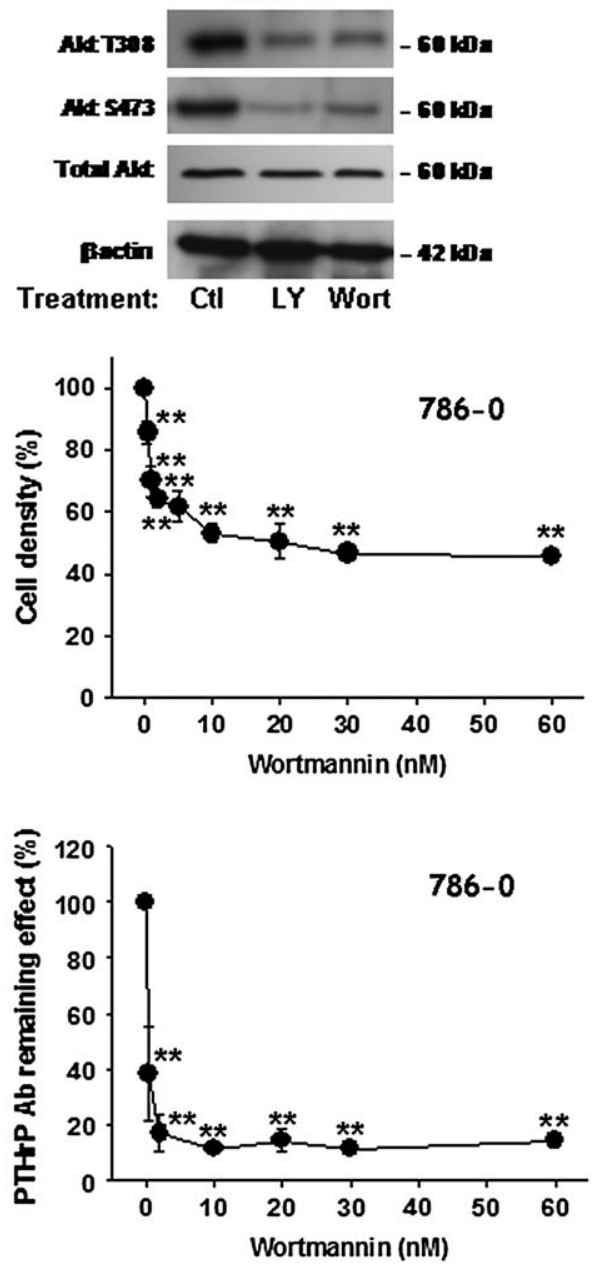

D

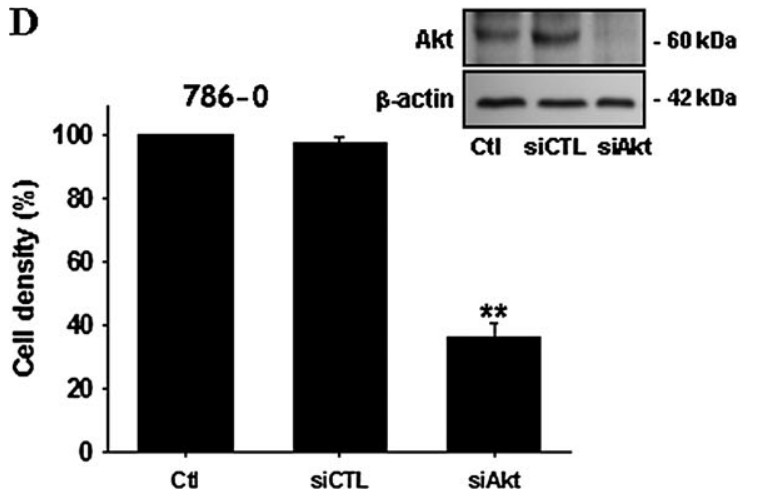

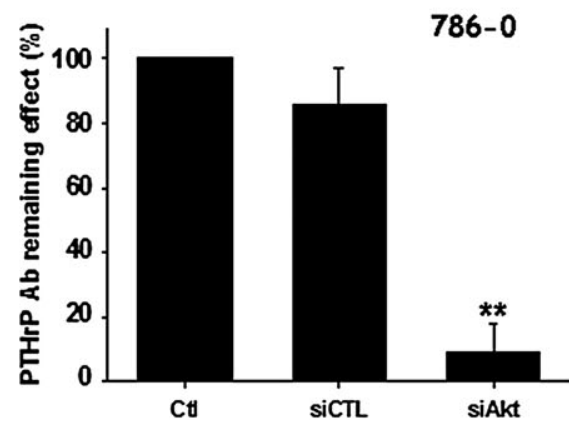

Fig. 2. PTHrP induces CRCC cells survival through activation of the PI $3 \mathrm{~K}-\mathrm{Akt}$-signaling pathway. (A) Immunoblots for unphosphorylated total Akt and phosphorylated Akt (T308 or S473) in 786-0 cells (left blots) and in Caki-1 cells (right blots) treated with LY294002 (20 $\mu$ M), wortmannin (20 nM) or in control $(\mathrm{Ctl})$. Western blot for $\beta$-actin are protein-loading controls. Shown are representative radiographs of at least three independent experiments. (B) Effects of LY294002 (left panel) and wortmannin (right panel) at various concentrations on cell density in 786-0 cells. Results are shown as mean \pm SEM, $n=7-15$ (left panel), $n=4$ (right panel) $;{ }^{*} P<0.05,{ }^{* *} P<0.01$ from no inhibitor. (C) Effects of LY294002 (left panel) and wortmannin (right panel) at various concentrations on the effect of PTHrP Ab (5 $\mu \mathrm{g} / \mathrm{ml})$ on cell density in 786-0 cells. Results are expressed as PTHrP Ab remaining effect. PTHrP Ab remaining effect represents the difference of the apoptotic effect of the PTHrP Ab alone with its effect in the presence of the inhibitor at various concentrations. The remaining effect is then expressed in percentage calculated from the effect of the PTHrP Ab alone. Results are shown as mean \pm SEM, $n=7-15$ (left panel), $n=4$ (right panel); ${ }^{*} P<$ $0.05,{ }^{* *} P<0.01$ from no inhibitor. (D) Effects of the transfection with Akt-specific siRNA (siAkt), control siRNA (siCTL) or no siRNA (Ctl, transfection reagent alone) on cell density (left panel) or PTHrP Ab remaining effect (right panel) in 786-0 cells. Cells were analyzed $96 \mathrm{~h}$ after transfection in the absence (left panel) or presence (right panel) of PTHrP Ab for $48 \mathrm{~h}$. As above, PTHrP Ab remaining effect represents the difference of the apoptotic effect of the PTHrP Ab alone with its effect in the presence of siCTL or siAkt. The remaining effect is then expressed in percentage calculated from the effect of the PTHrP Ab alone. Results are expressed as mean \pm SEM, $n=4$ (left panel), $n=4$ (right panel); ${ }^{* *} P<0.01$ from control. Inset: immunoblots for total Akt in cells transfected with siRNAs as indicated or in control. Shown is a representative radiograph of at least three independent transfection experiments. 
PTHrP blockade decreases specifically phosphorylation of Akt at S473

We then investigated the effect of PTHrP Ab on the phosphorylation status of Akt. In cells treated with PTHrP Ab for $24 \mathrm{~h}$, phosphorylation of Akt at T308 was not affected, but interestingly, phosphorylation at S473 was decreased compared with control cells treated with PTHrP $\mathrm{Ab}$ diluent (Figure 3A). Akt expression was similar in all experimental conditions. In these experiments, cells were also exposed to non-immune IgG to ensure that the effect observed on Akt phosphorylation was specific for PTHrP Ab (Figure 3A). In order to study the time dependency of the effect of PTHrP Ab on Akt phosphorylation, cells were exposed to PTHrP Ab for $0-48 \mathrm{~h}$. Phosphorylation of Akt at T308 was not affected at all time points in 786-0 cells (Figure 3B) and Caki-1 cells (Figure 3C). Phosphorylation of Akt at S473 decreased in a time-dependent manner in the presence of PTHrP Ab and was almost completely absent after $2-5 \mathrm{~h}$ in $786-0$ cells (Figure $3 \mathrm{~B}$ ) and already after 15 min in Caki-1 cells (Figure 3C). These effects were transient with a return to baseline levels after $24-48 \mathrm{~h}$. Thus, PTHrP $\mathrm{Ab}$ decreased Akt phosphorylation and activity by inhibiting specifically the phosphorylation of Akt at S473.

\section{Identification of ILK as a PDK-2}

PTHrP has been shown to regulate the proliferation of normal proximal tubular cells, from which CRCC originates, through PTH1Rdependent PKC and PKA activation. Thus, we first investigated whether these kinases may play a role as PDK-2 in human CRCC.
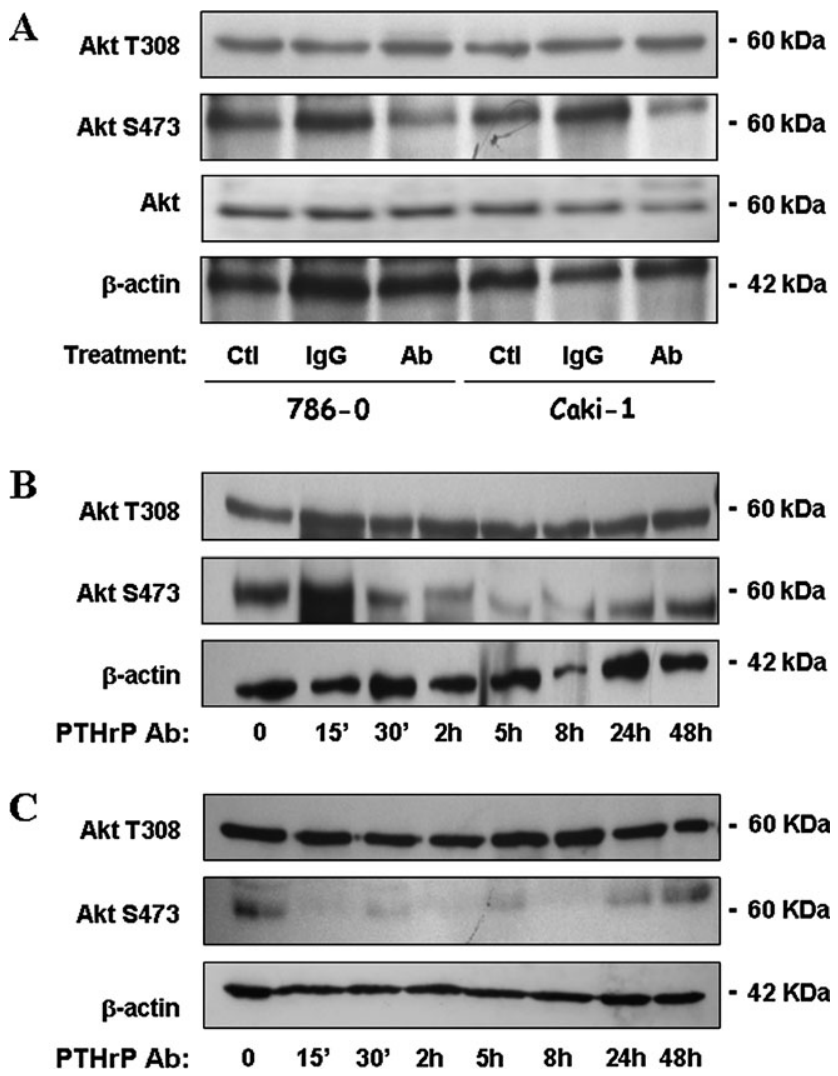

Fig. 3. PTHrP regulates Akt through specific phosphorylation at S473. (A) Immunoblots for unphosphorylated total Akt, phosphorylated Akt (T308), phosphorylated Akt (S473) and corresponding $\beta$-actin in 786-0 cells and in Caki-1 cells treated with non-immune IgG $(5 \mu \mathrm{g} / \mathrm{ml})$, PTHrP Ab $(5 \mu \mathrm{g} / \mathrm{ml})$ or in control $(\mathrm{Ctl})$. Shown are representative radiographs of at least three independent experiments. (B and C) Immunoblots for phosphorylated Akt (T308), Akt (S473) and corresponding $\beta$-actin in 786-0 (B) and Caki-1 (C) cells treated with PTHrP Ab for the time indicated in the figures. Shown are representative radiographs of at least three independent experiments for both cell types.
Using specific inhibitors and/or activators of PKC or Phospholipase $\mathrm{C}$ (PLC), we show that, although PKC is involved in the growth of human CRCC, it is not involved in PTHrP effect and does not have PDK-2 activity in human CRCC (data not shown). We obtained similar negative results in both cell lines when we investigated whether PKA or double-stranded DNA-dependent protein kinase act as PDK-2 with specific inhibitors (data not shown).

We followed this investigation by analyzing whether ILK is involved in PTHrP effect and whether it acts as a PDK-2 in human CRCC. Since similar results were obtained in both 786-0 and Caki1 cells, these investigations were performed in 786-0 cells. In a first set of experiments, CRCC cells were stably transfected with the various human ILK constructs described in Materials and methods. Seven clones of each transfection were picked-up and analyzed for ILK expression. Two clones of each transfection were then selected (data not shown) and the effects of the PTHrP Ab analyzed in all of them. No significant difference in the effects of PTHrP Ab $(48 \mathrm{~h}$ treatment) on cell density were observed in the clones compared with the effect in untransfected 786-0 cells or vector alone-transfected clones. No effects were observed in the clones obtained with the various constructs either alone or on the effect of the PTHrP Ab effect (data not shown). Transfection of 786-0 cells with ILK siRNA decreased ILK expression, as assessed $24 \mathrm{~h}$ after transfection, by $>50 \%$ and decreased phosphorylation of Akt at S473 without affecting phosphorylation at T308 (Figure 4, blot) indicating that ILK has PDK-2 activity in human CRCC. The transfection of ILK siRNA resulted in $30 \%$ decrease in cell density, as measured $96 \mathrm{~h}$ after transfection (Figure 4, top panel) and decreased PTHrP effect ( $48 \mathrm{~h}$ treatment) on cell density by $\sim 60 \%$ (Figure 4 , lower left panel). The effect of ILK-specific siRNA was obtained through induction of cell apoptosis, as shown by FACS analysis (Figure 4, lower right panel). No evidence of cell necrosis was observed in the presence of ILK-specific siRNA (data not shown). These results show that ILK contributes to the phosphorylation of Akt at S473 in human CRCC. They also indicate that ILK is involved in human CRCC growth not only by inhibiting tumor cell apoptosis but also by mediating PTHrP-induced tumor cell survival.

PTHrP induces cell survival through Akt-dependent $N F-\kappa B$ activation To identify downstream Akt targets regulated by PTHrP Ab, we performed western blot analyses in cells treated for $2 \mathrm{~h}$ with the PTHrP $\mathrm{Ab}$, with non-immune IgG or with the diluent; this time point corresponds to the maximal effect of PTHrP Ab on Akt phosphorylation (Figure 3B and C). The treatment with PTHrP Ab did not influence the phosphorylation status of GSK-3 $\beta$ and of cyclin D1 (at GSK-3 $\beta$ specific phosphorylation site T286) (data not shown). mTOR and FKHR were found to be expressed in both cell lines at the nonphosphorylated state and no effect of PTHrP Ab were observed on either their expression or their phosphorylation status (data not shown). Human non-small cell lung carcinoma of high-stage biopsy was used as a positive control to ensure that these phospho-specific Abs were working properly (data not shown). Another main downstream target of Akt is the pleiotropic transcription factor NF- $\mathrm{KB}$ $(12,13)$. NF- $\mathrm{KB}$ was found to be expressed and constitutively phosphorylated at the Akt-specific phosphorylation site (Figure 5A). The treatment of cells with PTHrP Ab resulted in a substantial decrease in $\mathrm{NF}-\kappa \mathrm{B}$ phosphorylation (Figure $5 \mathrm{~A}$ ). Consistent with this finding, the transient transfection of CRCC cells with ILK-specific siRNA decreased NF- $\kappa$ B phosphorylation (Figure 5A). Second, we measured the effect of a specific NF- $\kappa$ B inhibitor, BAY 11-7085 for $48 \mathrm{~h}$, either alone or in combination with PTHrP Ab. In 786-0 cells, the NF- $\mathrm{BB}$ inhibitor alone decreased cell density, an effect that was concentration dependent with $80 \%$ decrease at $5 \mu \mathrm{M}$ (Figure 5B, left panel). The importance of NF- $\mathrm{NB}$ in PTHrP-induced cell survival was confirmed by the decrease in PTHrP Ab effect in 786-0 cells in the presence of BAY 11-7085 (Figure 5B, right panel). Similar results were obtained in Caki-1 cells (data not shown). Finally, the transcriptional activity of $\mathrm{NF}-\kappa \mathrm{B}$ was directly measured in 786-0 cells treated with PTHrP Ab. The exposure of 786-0 cells to PTHrP Ab for 2 or $5 \mathrm{~h}$ decreased 

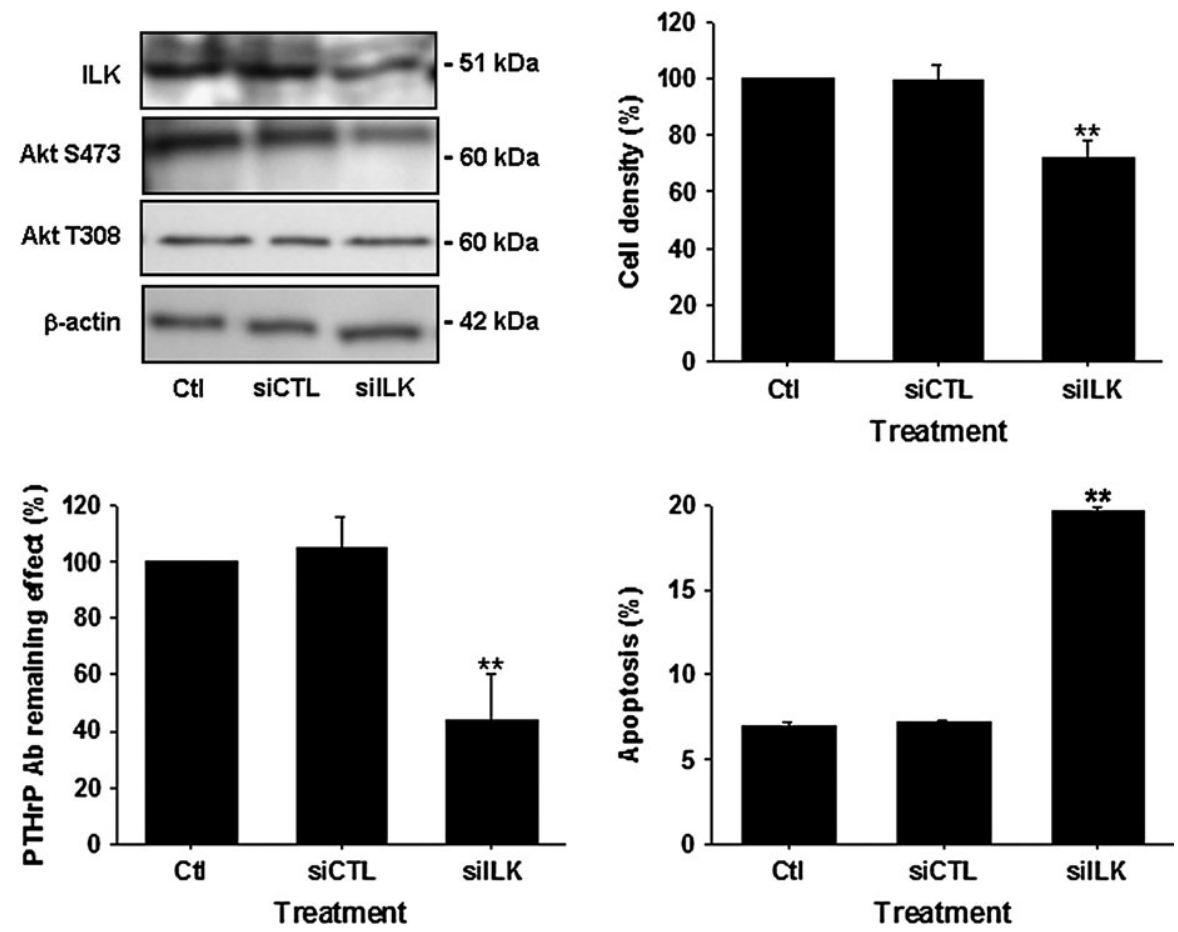

Fig. 4. PTHrP-induced Akt phosphorylation at S473 and tumor survival through ILK. Immunoblots for ILK, phosphorylated Akt (S473 and T308) and corresponding $\beta$-actin in 786-0 cells transfected with transfection reagent alone (control) or with control siRNA (siCTL) or ILK-specific siRNA (siILK) (top left). Effects of the transfection (Ctl, siCTL and siILK) on cell density in 786-0 cells (top right panel). Results are shown as mean \pm SEM, $n=4$; ${ }^{* *} P<0.01$ from control. Effects of the transfection (Ctl, siCTL and siILK) on the effect of PTHrP Ab $(5 \mu \mathrm{g} / \mathrm{ml})$ in 786-0 cells (lower left panel). Results are expressed as PTHrP Ab remaining effect. Again, PTHrP Ab remaining effect represents the difference of the apoptotic effect of the PTHrP Ab alone with its effect in the presence of siCTL or siILK. The remaining effect is then expressed in percentage calculated from the effect of the PTHrP Ab alone. Results are shown as mean \pm SEM, $n=4 ;{ }^{* *} P<$ 0.01 from control. Effects of the transfection (Ctl, siCTL and siILK) on cell apoptosis as assayed by FACS in 786-0 (lower right panel). Results are shown as mean \pm SEM, $n=8 ;{ }^{* *} P<0.01$ from control.

NF- $\kappa \mathrm{B}$ transcriptional activity by 20 and $40 \%$, respectively (Figure 5C). Similar results were obtained in Caki-1 cells (data not shown).

\section{Discussion}

We have recently demonstrated the crucial role played by PTHrP in the growth of human CRCC, independently of the VHL status of the cell $(10,11)$. As this type of tumor expresses high levels of PTHrP, exogenous addition of PTHrP did not affect tumor growth $(10,11)$. Therefore, the role of PTHrP/PTH1R system was evidenced using both PTHrP Abs and a potent PTH1R antagonist. However, the precise nature of the PTHrP-induced tumor survival effect was not completely investigated and the mechanism of this effect was not studied.

Here, we show that PTHrP induces tumor cell survival through inhibition of cell apoptosis and that the PI3K-Akt-signaling pathway is critical for this effect. We have shown previously that PTHrP is over-expressed in human CRCC $(10,11)$ and that Akt is constitutively activated in the same cancer (15). Within this context, these results suggest that PTHrP is involved in the constitutive phosphorylation/ activation of Akt kinase in human CRCC. Such role for PTHrP has never been described, although PTHrP has been shown to activate Akt pathway in other cells or tissues (20-22).

A very interesting finding in the present study was the observation that the treatment with PTHrP Ab completely and specifically abolished Akt phosphorylation at S473 suggesting that PTHrP is specifically involved in S473 phosphorylation in human CRCC. Again, such implication has never been described for PTHrP in any system. Although no data are available concerning such specific effects of any other factors in human $\mathrm{CRCC}$, this has been reported in other cell or tissue systems with different factors $(23,24)$. Our results suggest that PTHrP is necessary for full activity of Akt in human CRCC through activation of a PDK-2. Knowing its role, the identification of
PDK-2 is an important step toward the definition of new anticancer drugs.

We found that ILK acts as a PDK-2 or at least participates in the constitutive Akt phosphorylation at S473 in human CRCC. ILK is a serine/threonine kinase involved in cell survival, cell proliferation, cell migration and cell motility and has emerged as an important kinase involved in cancer development (25). Shortly after the discovery of PDK1, Dedhar et al. $(25,26)$ reported using multiple experimental approaches the identification of ILK as the kinase responsible for Akt phosphorylation at the hydrophobic motif domain. However, other cast doubt on whether ILK is a direct upstream kinase responsible for Akt phosphorylation at S473 (27-31). In addition, other studies, including in lower species, have shown that ILK regulates S473 phosphorylation by acting as an adaptor, either to recruit a distinct S473 kinase activity or to inhibit a S473-specific phosphatase activity $(27,32,33)$. Our results indicate that the level of ILK expression is high enough for its kinase activity not to be disturbed by transfection with inactive ILK proteins. Alternatively, its kinase activity may not be critical for its function but rather the level of its expression. ILK may then play the role of a facilitator protein and does not phosphorylate Akt directly in human CRCC; such assumptions will need additional experiments. We cannot rule out the possibility that additional kinases or proteins play this role in human CRCC, but clearly, these studies stress out the critical involvement of ILK in Akt phosphorylation at S473 and ultimately on human CRCC growth. The TOR kinase and its associated protein rictor have been shown to be necessary for Akt S473 phosphorylation and a reduction in rictor or mTOR expression inhibited an effector protein (34). However, in our previous studies (15) and in the present work, we did not observe any activation of mTOR in human CRCC, and the treatment with the PTHrP Ab did not affect the levels of mTOR protein. These studies suggest that the rictor-mTOR complex do 
A
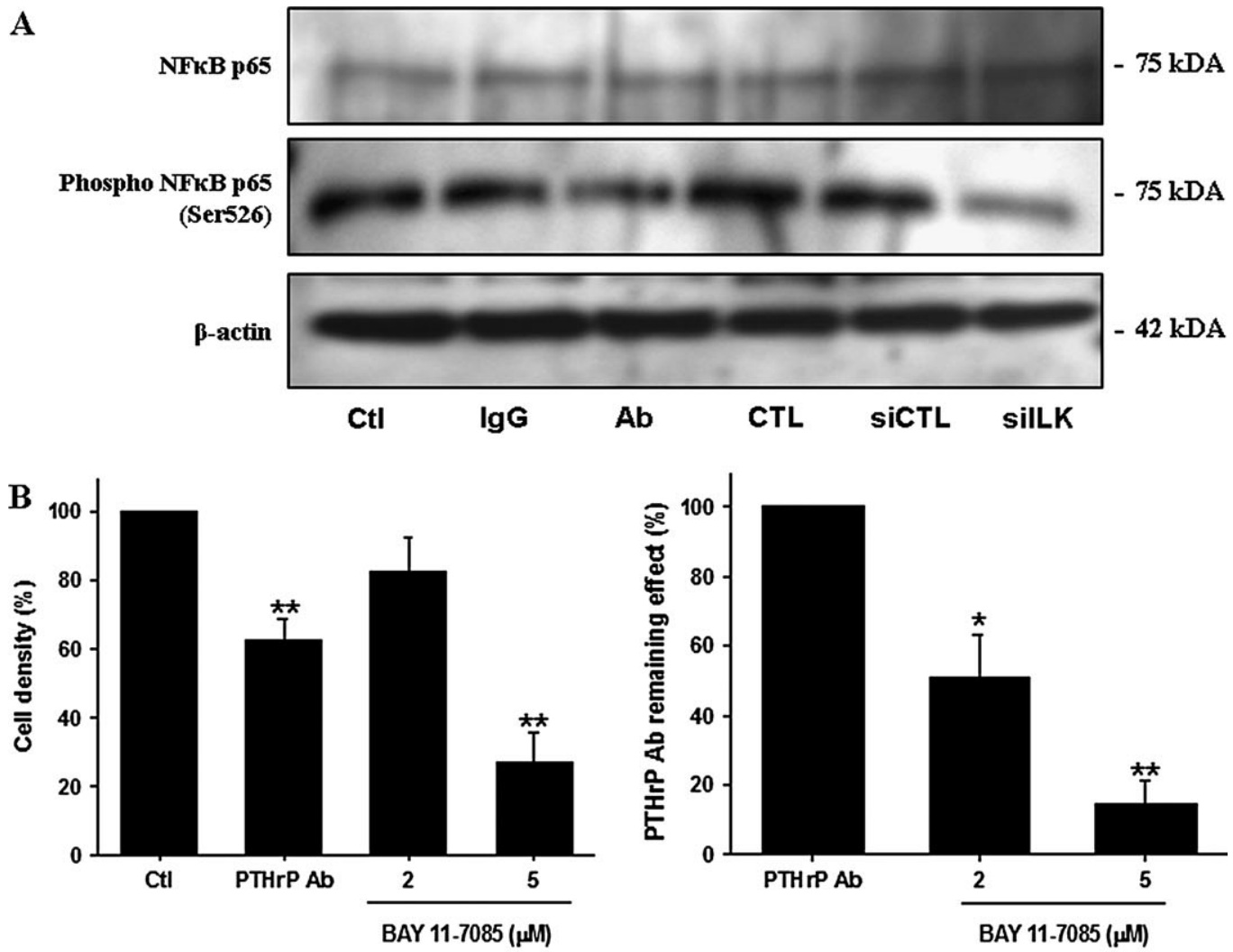

C

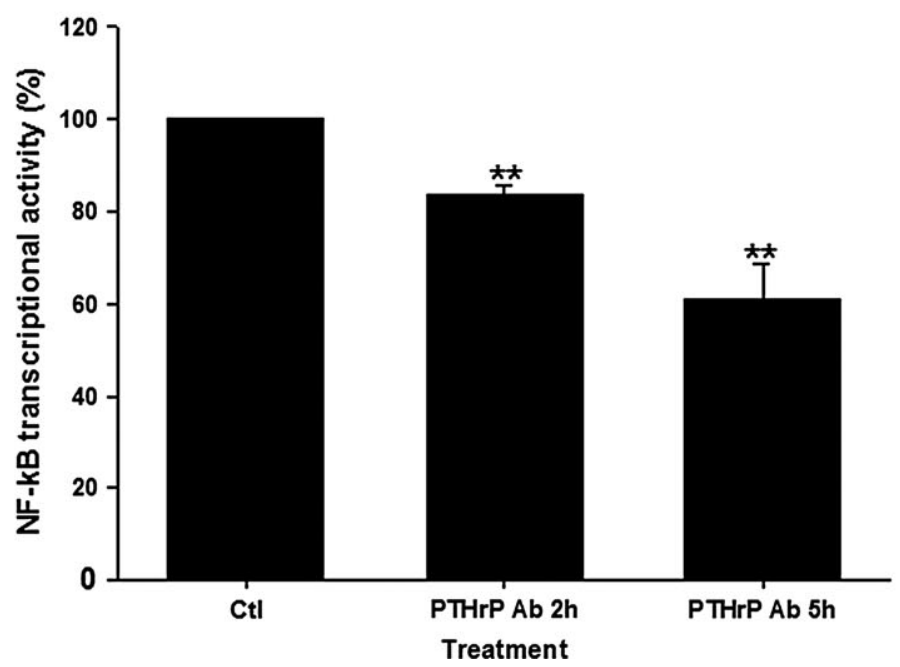

Fig. 5. PTHrP induced tumor cell survival through induction of ILK-dependent NF- $\kappa \mathrm{B}$ transcriptional activity. (A) Immunoblots for unphosphorylated and phosphorylated NF- $\kappa \mathrm{B}$ (S526) and corresponding $\beta$-actin in 786-0 cells treated for $2 \mathrm{~h}$ with non-immune IgG (5 $\mu \mathrm{g} / \mathrm{ml}), \mathrm{PTHrP}$ Ab (Ab, $5 \mu \mathrm{g} / \mathrm{ml})$, siCTL and silLK and corresponding controls (Ctl and CTL). (B) Effects of BAY 11-7085 (2 and $5 \mu \mathrm{M})$ on cell density (left panel) and on the effect of PTHrP Ab (5 $\mu \mathrm{g} / \mathrm{ml})$ in $786-0$ cells (right panel). Results are expressed as inhibitor effects (left panel) or as PTHrP Ab remaining effect (right panel). PTHrP Ab remaining effect represents the difference of the apoptotic effect of the PTHrP Ab alone with its effect in the presence of 2 or $5 \mu \mathrm{M}$ BAY 11-7085. The remaining effect is then expressed in percentage calculated from the effect of the PTHrP Ab alone. Results are shown as mean \pm SEM, $n=5 ;{ }^{*} P<0.05 ;{ }^{* *} P<0.01$ from control (left panel) or from PTHrP Ab effect (right panel). (C) Effect of PTHrP Ab on NF- $\kappa \mathrm{B}$ transcriptional activity. Results are expressed as mean \pm SEM, $n=3 ;{ }^{*} P<0.05,{ }^{* *} P<0.01$ from control.

not participate in the PDK-2 activity in human CRCC, although specific studies will be needed to ascertain this statement. Interestingly, we found GSK-3 constitutively phosphorylated in human CRCC as a consequence of the activation of Akt (15). Here, GSK-3 expression and phosphorylation were not found to be regulated by PTHrP. Although, this latter result may be related to ligand-specificity or to the cell compartmentalization of GSK-3, it has been shown recently that S473 phosphorylation affects only a subset of Akt substrates whereas others, such as GSK-3, are not affected (34).

The NF- $\kappa \mathrm{B}$ transcriptional pathway is involved in many biological processes including immunity, inflammation, angiogenesis, cell migration, cell proliferation and apoptosis (35). During its activation, $\mathrm{NF}-\kappa \mathrm{B}$ p65 undergoes phosphorylation, an important step for its biological activity. As for Akt, we found NF- $\kappa \mathrm{B}$ constitutively activated 


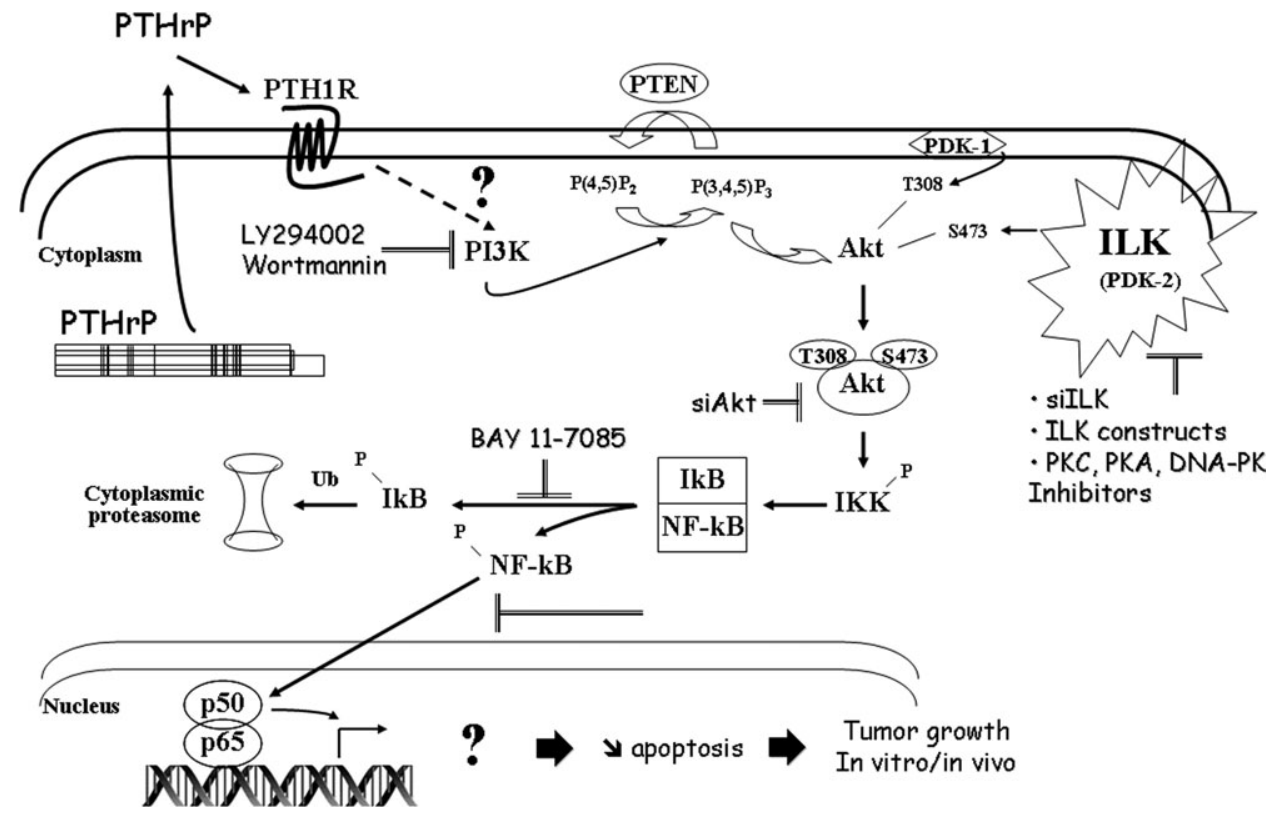

Fig. 6. Schematic representation of PTHrP signaling in human CRCC. PTHrP activates NF- $\kappa B$ through stimulation of ILK, identified as, at least, one of the kinase with PDK-2 activity in human CRCC, and Akt. The nature of PI3K activation by PTHrP is currently unknown but is probably PTH1R-dependent (31). $\mathrm{P}(4,5) \mathrm{P} 2$ : phosphatidylinositol-4,5-bisphosphate; $\mathrm{P}(3,4,5) \mathrm{P} 3$ : phosphatidylinositol-3,4,5-trisphosphate; PTEN: phosphatase and tensin homologue deleted on chromosome 10 and MCP: monocyte chemoattractant protein. Experimental tools used in the present study are shown.

in both 786-0 and Caki-1 cells, consistent with previous studies (36-39). The possible involvement of NF- $\mathrm{KB}$ in human CRCC tumorigenesis has only received little attention and the few data available on cultured cells are in most cases difficult to interpret or contradictory $(36,38-41)$. Using a specific NF- $\mathrm{KB}$ inhibitor and transcription assay, we show that NF- $\kappa \mathrm{B}$ is involved in human $\mathrm{CRCC}$ growth and that it is critical for the survival effect of PTHrP. In addition, we also linked ILK expression, Akt activation and NF- $\kappa B$ activity in human CRCC. PTHrP has been shown to stimulate NF- $\kappa \mathrm{B}$ or not depending on the cell or tissue considered (42-44). In their report, Guillen et al. (42) showed that PTHrP activates NF- $\kappa \mathrm{B}$ leading to an increase in IL-6 expression. IL- 6 has been shown to be expressed in human CRCC and to be involved in tumorigenesis through inhibition of tumor cell apoptosis (45). Thus, whether PTHrP stimulates IL-6 expression and activity through NF- $\kappa \mathrm{B}$ remains an open and interesting question for future investigations.

Interestingly, we observed that the decrease of Akt phosphorylation is faster in Caki-1 cells compared with 786- 0 cells (i.e. 15 min versus $2-5 \mathrm{~h}$ ) in response to PTHrP Ab exposure (Figure 3 ). This could be the consequence of the VHL status of the cells or to differences in cell types. To ascertain one of these possibilities, further studies using VHL-deficient cells transfected with VHL constructs will be needed. In accordance with our results, the loss of VHL has been shown to heighten NF- $\kappa \mathrm{B}$ activity in human $\mathrm{CRCC}$ through hypoxia-induced transcription factor induced increase of transforming growth factor $\alpha$ expression and subsequent activation of epidermal growth factor receptor/Akt/NF- $\kappa \mathrm{B}$-signaling pathway (39).

Taken together, our results show that PTHrP, by stimulating ILK identified as a kinase with PDK-2 activity, participates in the constitutive activation of the PI3K/Akt/NF- $\mathrm{KB}$-signaling pathway and induces tumor cell survival (Figure 6). The question of how PTHrP stimulates PI3K remains open. The possibility that the PTH1R is coupled to PI3K has never been explored. The fact that PKA and PKC inhibitors did not influence PTHrP activity suggest that in contrast to other cells and tissues, including proximal tubular cells from which CRCC originates, PTH1R is not coupled to adenylyl cyclase or PLC in malignant tubular cells. A further understanding of PTHrPdependent NF- $\kappa \mathrm{B}$ target genes in human CRCC may prove critical for the definition of new and potent therapeutics against this refractory disease. In addition, targeting ILK, which acts as an upstream protein involved in constitutive Akt and NF- $\kappa \mathrm{B}$ activations, may also have therapeutic potential as shown for other extra-renal malignancies.

\section{Funding}

INSERM to J.J.H.; University Louis Pasteur of Strasbourg, Strasbourg School of Medicine to T.M.; the French Ligue Contre le Cancer Comités du Bas-Rhin et du Haut-Rhin et Comité National to T.M.; Association pour la Recherche sur le Cancer to T.M.

\section{Acknowledgements}

We thank Ms F.Thirion and F.Reymann for technical assistance in immunohistochemistry studies.

Conflict of Interest statement: None declared.

\section{References}

1. Curti,B.D. (2004) Renal cell carcinoma. JAMA, 292, 97-100.

2. Cohen,H.T. (2005) Renal cell carcinoma. N. Engl. J. Med., 353, 24772490.

3. Sourbier,C. et al. (2006) Parathyroid hormone-related protein in human renal cell carcinoma. Cancer Lett., 240, 170-182.

4. Latif,F. et al. (1993) Identification of the von Hippel-Lindau disease tumor suppressor gene. Science, 260, 1317-1320.

5. Gnarra,J.R. et al. (1994) Mutations of the VHL tumour suppressor gene in renal carcinoma. Nat. Genet., 7, 85-90.

6. Friedrich,C.A. (1999) Von Hippel-Lindau syndrome. A pleomorphic condition. Cancer, 86, 2478-2482.

7. Wiesener,M.S. et al. (2001) Constitutive activation of hypoxia-inducible genes related to overexpression of hypoxia-inducible factor- 1 in clear cell renal carcinomas. Cancer Res., 61, 5215-5222.

8. Kim,W. et al. (2003) The von Hippel-Lindau tumor suppressor protein: new insights into oxygen sensing and cancer. Curr. Opin. Genet. Dev., 13, 55-60.

9. Esbrit,P. et al. (2000) The emerging role of parathyroid hormone-related protein as a renal regulating factor. Nephrol. Dial. Transplant., 15, 1109 1111. 
10. Massfelder,T. et al. (2004) Parathyroid hormone-related protein is an essential growth factor for human clear cell renal carcinoma and a target for the von Hippel-Lindau tumor suppressor gene. Cancer Res., 64, 180-188.

11. Talon,I. et al. (2006) Anti-tumor effect of parathyroid hormone-related protein neutralizing antibody in human renal cell carcinoma in vitro and in vivo. Carcinogenesis, 27, 73-83.

12. Nicholson,K.M. et al. (2002) The protein kinase B/Akt signalling pathway in human malignancy. Cell. Signal., 14, 381-395.

13. Hanada,M. et al. (2004) Structure, regulation and function of PKB/ AKT - a major therapeutic target. Biochem. Biophys. Acta, 1697, 3-16.

14. Dong,L.Q. et al. (2005) PDK2: the missing piece in the receptor tyrosine kinase signaling pathway puzzle. Am. J. Physiol. Endocrinol. Metab., 289, E187-E1196.

15. Sourbier,C. et al. (2006) The phosphoinositide 3-kinase/Akt pathway: a new target in human renal cell carcinoma therapy. Cancer Res., 66 , $5130-5142$.

16. Iliopoulos, O. et al. (1995) Tumour suppression by the human von HippelLindau gene product. Nat. Med., 1, 822-826.

17. Lowry,O.H. et al. (1951) Protein measurement with the Folin phenol reagent. J. Biol. Chem., 193, 265-275.

18. Fresno Vara,J.A. et al. (2004) PI3K/Akt signalling pathway and cancer Cancer Treat. Rev., 30, 193-204.

19. Skinner,H.D. et al. (2004) Vascular endothelial growth factor transcriptional activation is mediated by hypoxia-inducible factor 1alpha, HDM2, and p70S6K1 in response to phosphatidylinositol 3-kinase/AKT signaling. J. Biol. Chem., 279, 45643-45651.

20. Chen,H.L. et al. (2002) Parathyroid hormone and parathyroid hormonerelated protein exert both pro- and anti-apoptotic effects in mesenchymal cells. J. Biol. Chem., 277, 19374-19381.

21. Grzesiak,J.J. et al. (2005) GSK3 and PKB/Akt are associated with integrinmediated regulation of PTHrP, IL-6 and IL-8 expression in FG pancreatic cancer cells. Int. J. Cancer, 114, 522-530.

22. Ortega,A. et al. (2006) Role of parathyroid hormone-related protein in tubulointerstitial apoptosis and fibrosis after folic acid-induced nephrotoxicity. J. Am. Soc. Nephrol., 17, 1594-1603.

23. O'toole,A. et al. (2001) Tumour necrosis factor-alpha activation of protein kinase B in WEHI-164 cells is accompanied by increased phosphorylation of Ser473, but not Thr308. Biochem. J., 359, 119-127.

24. Weigert,C. et al. (2005) Interleukin-6 acts as insulin sensitizer on glycogen synthesis in human skeletal muscle cells by phosphorylation of Ser473 of Akt. Am. J. Physiol. Endocrinol. Metab., 289, E251-E257.

25. Hannigan,G. et al. (2005) Integrin-linked kinase: a cancer therapeutic target unique among its ILK. Nat. Rev. Cancer, 5, 51-63.

26. Persad,S. et al. (2001) Regulation of protein kinase B/Akt-serine 473 phosphorylation by integrin-linked kinase: critical roles for kinase activity and amino acids arginine 211 and serine 343. J. Biol. Chem., 276, 27462-27469.

27. Lynch,D.K. et al. (1999) Integrin-linked kinase regulates phosphorylation of serine 473 of protein kinase B by an indirect mechanism. Oncogene, $\mathbf{1 8}$ 8024-8032.

28. Chan,T.O. et al. (2001) PDK2: a complex tail in one Akt. Sci. STKE, 66, PE1.
29. Hill,M.M. et al. (2002) Identification of a plasma membrane Raftassociated PKB Ser473 kinase activity that is distinct from ILK and PDK1. Curr. Biol., 12, 1251-1255.

30. Grashoff,C. et al. (2003) Integrin-linked kinase regulates chondrocyte shape and proliferation. EMBO Rep., 4, 432-443.

31. Sakai,T. et al. (2003) Integrin-linked kinase (ILK) is required for polarizing the epiblast, cell adhesion, and controlling actin accumulation. Genes Dev., 17, 926-940.

32.Zervas,C.G. et al. (2001) Drosophila integrin-linked kinase is required at sites of integrin adhesion to link the cytoskeleton to the plasma membrane. J. Cell Biol., 152, 1007-1018.

33. Mackinnon,A.C. et al. (2002) C. elegans PAT-4/ILK functions as an adaptor protein within integrin adhesion complexes. Curr. Biol., 12, 787-797.

34. Jacinto,E. et al. (2006) SIN1/MIP1 maintains rictor-mTOR complex integrity and regulates Akt phosphorylation and substrate specificity. Cell, 127, 125-137.

35. Hayden,M.S. et al. (2004) Signaling to NF-kappaB. Genes Dev., 18, 2195 2224

36. Oya,M. et al. (2001) Constitutive activation of nuclear factor-kappaB prevents TRAIL-induced apoptosis in renal cancer cells. Oncogene, 20, 3888-3896.

37. Oya,M. et al. (2003) Increased nuclear factor-kappa B activation is related to the tumor development of renal cell carcinoma. Carcinogenesis, 24, 377-384.

38. An,J. et al. (2005) VHL expression in renal cell carcinoma sensitizes to bortezomib (PS-341) through an NF-kappaB-dependent mechanism. Oncogene, 24, 1563-1570.

39. An,J. et al. (2005) Mechanism of von Hippel-Lindau protein-mediated suppression of nuclear factor kappa B activity. Mol. Cell. Biol., 25 , 7546-7556

40. Pawlowski,J.E. et al. (2000) NF-kappa B does not modulate sensitivity of renal carcinoma cells to TNF alpha-related apoptosis-inducing ligand (TRAIL). Anticancer Res., 20, 4243-4255.

41. An,J. et al. (2004) Maximal apoptosis of renal cell carcinoma by the proteasome inhibitor bortezomib is nuclear factor-kappaB dependent. Mol. Cancer Ther, 3, 727-736.

42. Guillen,C. et al. (2002) Both N- and C-terminal domains of parathyroid hormone-related protein increase interleukin- 6 by nuclear factor-kappa B activation in osteoblastic cells. J. Biol. Chem., 277, 28109-28117.

43.Zuscik,M.J. et al. (2002) Lead alters parathyroid hormone-related peptide and transforming growth factor-beta1 effects and AP-1 and NF-kappaB signaling in chondrocytes. J. Orthop. Res., 20, 811-818.

44. Martin-Ventura,J.L. et al. (2003) Possible role of parathyroid hormonerelated protein as a proinflammatory cytokine in atherosclerosis. Stroke, 34, 1783-1789.

45. Ueno,S. et al. (2006) Plasma membrane-associated sialidase is upregulated in renal cell carcinoma and promotes interleukin-6-induced apoptosis suppression and cell motility. J. Biol. Chem., 281, 7756-7764.

Received January 29, 2007; revised April 2, 2007; accepted April 24, 2007 